\title{
Throughput Analysis of a Probabilistic Topology-Unaware TDMA MAC Policy for Ad-Hoc Networks
}

\author{
Konstantinos Oikonomou ${ }^{1}$ and Ioannis Stavrakakis ${ }^{2}$ \\ 1 INTRACOM S.A., Development Programmes Department, \\ 19.5 Markopoulou Avenue, Paiania 19002 \\ Athens, Greece \\ Tel: +30210 6677023, Fax: +302106671312 \\ okon@intracom.gr \\ 2 University of Athens, Department of Informatics \& Telecommunications \\ Panepistimiopolis, Ilissia 15784 \\ Athens, Greece \\ Tel: +30 2107275343 , Fax: +30 2107275333 \\ istavrak@di.uoa.gr
}

\begin{abstract}
The design of an efficient Medium Access Control (MAC) is challenging in ad-hoc networks where users can enter, leave or move inside the network without any need for prior configuration. The existing topology-unaware TDMA-based schemes are capable of providing a minimum guaranteed throughput by considering a deterministic policy for the utilization of the assigned scheduling time slots. In an earlier work, a probabilistic policy that utilizes the non-assigned slots according to an access probability, common for all nodes in the network, was proposed. The achievable throughput for a specific transmission under this policy was analyzed. In this work, the system throughput is studied and the conditions under which the system throughput under the probabilistic policy is higher than that under the deterministic policy are established. In addition, the value for the access probability that maximizes the system throughput is determined analytically, as well as simplified lower and upper bounds that depend only on a topology density metric. Since the analysis of the system throughput is shown to be difficult or impossible in the general case an approximation is introduced whose accuracy is investigated. Simulation results show that the approximate analysis successfully determines the range of values for the access probability for which the system throughput under the probabilistic policy is not only higher than that under the deterministic, but it is also close to the maximum.
\end{abstract}

\section{Introduction}

Ad-hoc networks require no infrastructure and nodes are free to enter, leave or move inside the network without prior configuration. This flexibility introduces new challenges and makes the design of an efficient Medium Access Control (MAC) a challenging problem. CSMA/CA-based MAC protocols have been proposed, [1], [2], [3], whereas others have employed handshake mechanisms like the Ready-To-Send/Clear-To-Send (RTS/CTS) mechanism [4], [5], to avoid the hidden/exposed terminal problem.

Chlamtac and Farago, [6], have proposed an original TDMA-based scheme for topology transparent scheduling. The mathematical properties of polynomials with coefficients from finite Galois fields are exploited to randomly assign scheduling time slot sets to each node of the network. It is guaranteed that for each node at least one time slot in a frame would be collision-free, [6]. Another scheme proposed by $\mathrm{Ju}$ and $\mathrm{Li}$, [7], maximizes the minimum guaranteed throughput. However, both schemes employ a deterministic policy for the utilization of the assigned time slots that fails to utilize non-assigned time slots that could result in successful transmissions, as it is shown here.

In a previous work, [8], the general approach proposed in [6] and [7] (to be referred to as the Deterministic Policy) was considered and the idea of allowing the nodes to utilize (according to a common access probability) scheduling slots not originally assigned (according to the rules in [6], [7]) to them, was introduced. The proposed policy (to be referred to as the Probabilistic Policy) was analyzed for a specific transmission and the conditions under which the throughput for a specific transmission under the Probabilistic Policy is higher than that under the Deterministic Policy, were established. In addition, it was shown that for "large networks" there is always a efficient range of values for the access probability (a range of values such that the Probabilistic Policy outperforms the Deterministic). Simulation results had shown how the topology density affects the size of the efficient range of values. In any case, the particular work did not determine the maximum value of the system throughput and the corresponding value for the access probability.

In this work, the system throughput under the Probabilistic Policy is analyzed extensively and the conditions for the existence of an efficient range of values for the access probability are established, based on a properly defined topology density metric. In addition, this analysis determines the maximum value for the system throughput and the value for the 
access probability that achieves it. Simple lower and upper bounds for this value that depend only on a simple topology density metric are also determined. Part of the previous analysis is based on approximations since the exact analysis of the system throughput appeared to be difficult or impossible in the general case. This approximate analysis assumes that the number of neighbor nodes is the same for all nodes in the network and equal to the average number of neighbor nodes. It turns out that this approximation leads to fairly accurate results under certain conditions that are studied here.

In Section 2, a general ad-hoc network is described as well as both the Probabilistic Policy and the Deterministic Policy. In Section 3, the preliminary system throughput analysis shows that it is difficult or impossible, in the general case, to fully analyze it. An approximate analysis is presented in Section 4 that establishes the conditions for the existence of an efficient range of values for the access probability. Furthermore, this analysis determines the maximum value for the system throughput and the corresponding value for the access probability; bounds on the latter probability are determined analytically as a function of the topology density. In Section 5, the accuracy of the approximate analysis is studied. Simulation results, presented in Section 6, show that a value for the access probability that falls within the bounds, as they are determined based on the topology density, results in a system throughput that is close to the maximum. Section 7 presents the conclusions.

\section{Scheduling Policies}

An ad-hoc network may be viewed as a time varying multihop network and may be described in terms of a graph $G(V, E)$, where $V$ denotes the set of nodes and $E$ the set of links between the nodes at a given time instance. Let $|X|$ denote the number of elements in set $X$ and let $N=|V|$ denote the number of nodes in the network. Let $S_{u}$ denote the set of neighbors of node $u, u \in V$. These are the nodes $v$ to which a direct transmission from node $u$ (transmission $u \rightarrow v)$ is possible. Let $D$ denote the maximum number of neighbors for a node; clearly $\left|S_{u}\right| \leq D, \forall u \in V$.

Suppose that a node $u$ wants to transmit to a particular neighbor node $v$ in a particular time slot $i$. In order for the transmission $u \rightarrow v$ to be successful, two conditions should be satisfied. First, node $v$ should not transmit in the particular time slot $i$, or equivalently, no transmission $v \rightarrow \psi, \forall \psi \in S_{v}$ should take place in time slot $i$. Second, no neighbor of $v$ - except $u$ - should transmit in time slot $i$, or equivalently, no transmission $\zeta \rightarrow \chi, \forall \zeta \in S_{v}-\{u\}$ and $\chi \in S_{\zeta}$, should take place in time slot $i$. Consequently, transmission $u \rightarrow v$ is corrupted in time slot $i$ if at least one transmission $\chi \rightarrow \psi, \chi \in S_{v} \cup\{v\}-\{u\}$ and $\psi \in S_{\chi}$, takes place in time slot $i$.

The transmission(s) that corrupts transmission $u \rightarrow v$ may or may not be successful itself. Specifically, in the presence of transmission $u \rightarrow v$, transmission $\chi \rightarrow \psi, \chi \in S_{v} \cup\{v\}-\{u\}$ and $\psi \in S_{\chi} \cap\left(S_{u} \cup\{u\}\right)$, is corrupted. If $\psi \in$ $S_{\chi}-\left(S_{\chi} \cap\left(S_{u} \cup\{u\}\right)\right)$, then transmission $\chi \rightarrow \psi$ is not affected by transmission $u \rightarrow v$.

Under the Deterministic Policy, [6], [7], each node $u \in V$ is randomly assigned a unique polynomial $f_{u}$ of degree $k$ with coefficients from a finite Galois field of order $q(G F(q))$. Polynomial $f_{u}$ is represented as $f_{u}(x)=\sum_{i=0}^{k} a_{i} x^{i}(\bmod q)$ [7], where $a_{i} \in\{0,1,2, \ldots, q-1\}$; parameters $q$ and $k$ are calculated based on $N$ and $D$, according to the algorithm presented either in [6] or [7]. For both algorithms it is satisfied that $k \geq 1$ and $q \geq k D+1$ ( $k$ and $D$ are integers).

The access scheme considered is a TDMA scheme with a frame consisted of $q^{2}$ time slots. If the frame is divided into $q$ subframes $s$ of size $q$, then the time slot assigned to node $u$ in subframe $s,(s=0,1, \ldots, q-1)$ is given by $f_{u}(s)$ mod $q$ [7]. Let the set of time slots assigned to node $u$ be denoted as $\Omega_{u}$. Clearly, $\left|\Omega_{u}\right|=q$. The deterministic transmission policy, [6], [7], is the following.

The Deterministic Policy: Each node $u$ transmits in a slot $i$ only if $i \in \Omega_{u}$, provided that it has data to transmit.

Depending on the particular random assignment of the polynomials, it is possible that two nodes be assigned overlapping time slots (i.e., $\Omega_{u} \cap \Omega_{v} \neq \emptyset$ ). Let $C_{u \rightarrow v}$ be the set of overlapping time slots between those assigned to node $u$ and those assigned to any node $\chi \in S_{v} \cup\{v\}-\{u\} . C_{u \rightarrow v}$ is given by:

$$
C_{u \rightarrow v}=\Omega_{u} \cap\left(\bigcup_{\chi \in S_{v} \cup\{v\}-\{u\}} \Omega_{\chi}\right) .
$$

Let $R_{u \rightarrow v}$ denote the set of time slots $i, i \notin \Omega_{u}$, over which transmission $u \rightarrow v$ would be successful. Equivalently, $R_{u \rightarrow v}$ contains those slots not included in set $\bigcup_{\chi \in S_{v} \cup\{v\}} \Omega_{\chi}$. Consequently,

$$
\left|R_{u \rightarrow v}\right|=q^{2}-\left|\bigcup_{\chi \in S_{v} \cup\{v\}} \Omega_{\chi}\right|
$$


$R_{u \rightarrow v}$ is the set of non-assigned eligible time slots for transmission $u \rightarrow v$; if such slots are used by transmission $u \rightarrow v$, the probability of success for the particular transmission could be increased. The increased probability of success for transmission $u \rightarrow v$ does not necessarily increase the average probability of success of all transmissions in the network (system throughput); the presence of transmission $u \rightarrow v$ in a slot $i, i \notin \Omega_{u}$, may corrupt another, otherwise successful, transmission $\chi \rightarrow \psi$, even though transmission $u \rightarrow v$ will be successful.

Theorem 1. $\left|R_{u \rightarrow v}\right|$ is greater than or equal to $q(k-1) D$.

The proof of Theorem 1 can be found in Appendix 1.

From Theorem 1 it is obvious that for $k>1,\left|R_{u \rightarrow v}\right|>q D$. Consequently, the number of non-assigned eligible slots may be quite significant for the cases where $k>1$ (this case corresponds to large networks, [7]). Even for the case where $k=1,\left|R_{u \rightarrow v}\right| \geq 0$, that is, $\left|R_{u \rightarrow v}\right|$ can still be greater than zero. For those nodes for which the set of overlapping slots is not the largest possible (i.e., $\left.\left|\bigcup_{\chi \in S_{v} \cup\{v\}} \Omega_{\chi}\right|<\left(\left|S_{v}\right|+1\right) q\right),\left|R_{u \rightarrow v}\right|$ is greater than zero, even for $k=1$. Furthermore, if the neighborhood of node $v$ is not dense, or $\left|S_{v}\right|$ is small compared to $D$, then $\left|R_{u \rightarrow v}\right|$ is even higher.

In general, the use of slots $i, i \in R_{u \rightarrow v}$, may increase the average number of successful transmissions, as long as $R_{u \rightarrow v}$ is determined and time slots $i \in R_{u \rightarrow v}$ are used efficiently. The determination of $R_{u \rightarrow v}$ requires the existence of a mechanism for the extraction of sets $\Omega_{\chi}, \forall \chi \in S_{v}$. In addition, the efficient use of slots in $R_{u \rightarrow v}$ by node $u$, requires further coordination and control exchange with neighbor nodes $\chi$, whose transmissions $\chi \rightarrow \psi$, with $R_{\chi \rightarrow \psi} \cap R_{u \rightarrow v} \neq \emptyset$, may utilize the same slots in $R_{\chi \rightarrow \psi} \cap R_{u \rightarrow v}$ and corrupt either transmission $u \rightarrow v$ or $\chi \rightarrow \psi$, or both.

Moreover, under non-heavy traffic conditions, there exist a number of idle slots, in addition to those in $R_{u \rightarrow v}$, not used by the node they are assigned to. In order to use all non-assigned time slots without the need for further coordination among the nodes, the following probabilistic transmission policy is proposed.

The Probabilistic Policy: Each node $u$ always transmits in slot $i$ if $i \in \Omega_{u}$ and transmits with probability $p$ in slot $i$ if $i \notin \Omega_{u}$, provided it has data to transmit.

The Probabilistic Policy does not require specific topology information (e.g., knowledge of $R_{u \rightarrow v}$, etc.) and, thus, induces no additional control overhead. The access probability $p$ is a simple parameter common for all nodes. Under the Probabilistic Policy, all slots $i \notin \Omega_{u}$ are potentially utilized by node $u$ : both, those in $R_{u \rightarrow v}$, for a given transmission $u \rightarrow v$, as well as those not in $\Omega_{u} \cup R_{u \rightarrow v}$ that may be left by neighboring nodes under non-heavy traffic conditions. On the other hand, the probabilistic transmission attempts induce interference to otherwise collision-free transmissions. The following section establishes the conditions under which the loss due to the induced interference is more than compensated for by the utilization of the non-assigned time slots.

\section{System Throughput}

The throughput for a specific transmission under the Probabilistic and Deterministic policies was investigated in [8]. In this section the expressions for the system throughput under both policies are provided and the conditions under which the Probabilistic Policy outperforms the Deterministic Policy are derived. When these conditions are satisfied it is shown that there exists an efficient range of values for $p$ (such that the system throughput under the Probabilistic Policy is higher than that under the Deterministic Policy). The analysis assumes heavy traffic conditions; that is, there is always data available for transmission at each node, for every time slot.

Let $P_{i, u \rightarrow v}$ denote the probability that transmission $u \rightarrow v$ in slot $i$ is successful. Under the Deterministic Policy, $P_{i, u \rightarrow v}=0, \forall i \notin \Omega_{u}$. For $i \in \Omega_{u}$ there are two distinct cases: for $i \in C_{u \rightarrow v}, P_{i, u \rightarrow v}=0$, while for $i \notin C_{u \rightarrow v}, P_{i, u \rightarrow v}=1$ (note from Equation (1) that if $i \in C_{u \rightarrow v}$ then $i \in \Omega_{u}$ ). Since $\left|\Omega_{u}\right|=q$, it is evident that under the Deterministic Policy the average over a frame probability of success for transmission $u \rightarrow v$ (denoted by $P_{D, u \rightarrow v}$ ), is given by

$$
P_{D, u \rightarrow v}=\frac{q-\left|C_{u \rightarrow v}\right|}{q^{2}},
$$

where $q^{2}$ is the frame size in time slots.

Under the Probabilistic Policy, it is evident that $P_{i, u \rightarrow v}=0$, for $i \in C_{u \rightarrow v}$, as well as for $i \notin \Omega_{u}$ and $i \notin R_{u \rightarrow v}$. On the other hand, $P_{i, u \rightarrow v}=(1-p)^{\left|S_{v}\right|}$, for $i \in \Omega_{u}$ and $i \notin C_{u \rightarrow v}$, whereas $P_{i, u \rightarrow v}=p(1-p)^{\left|S_{v}\right|}$, for $i \in R_{u \rightarrow v}$ (note from Equation (2) that if $i \in R_{u \rightarrow v}$ then $i \notin \Omega_{u}$ ). Consequently, $P_{i, u \rightarrow v}=(1-p)^{\left|S_{v}\right|}$ for $q-\left|C_{u \rightarrow v}\right|$ time slots, while $P_{i, u \rightarrow v}=p(1-p)^{\left|S_{v}\right|}$ for $\left|R_{u \rightarrow v}\right|$ time slots. As a result, under the Probabilistic Policy the average over a frame probability of success for transmission $u \rightarrow v$ (denoted by $P_{P, u \rightarrow v}$ ), is given by

$$
P_{P, u \rightarrow v}=\frac{q-\left|C_{u \rightarrow v}\right|+p\left|R_{u \rightarrow v}\right|}{q^{2}}(1-p)^{\left|S_{v}\right|} .
$$


Let $P_{D, \text { succ }}\left(P_{P, \text { succ }}\right)$ denote the probability of success of a transmission (averaged over all transmissions) under the Deterministic (Probabilistic) Policy (be referred to as the system throughput for both policies) assuming that each node $u$ may transmit to only one node $v \in S_{u}$ in one frame. $P_{D, s u c c}$ and $P_{P, s u c c}$ are given by the following equations.

$$
\begin{aligned}
P_{D, \text { succ }} & =\frac{1}{N} \sum_{\forall u \in V} \frac{q-\left|C_{u \rightarrow v}\right|}{q^{2}}, \\
P_{P, \text { succ }} & =\frac{1}{N} \sum_{\forall u \in V} \frac{q-\left|C_{u \rightarrow v}\right|+p\left|R_{u \rightarrow v}\right|}{q^{2}}(1-p)^{\left|S_{v}\right|},
\end{aligned}
$$

where $v \in S_{u}$. From Equation (6) it can be seen that for $p=0, P_{P, \text { succ }}=P_{D, s u c c}$, while for $p=1, P_{P, s u c c}=0$. In general, $P_{P, \text { succ }}$ may or may not be greater than $P_{D, \text { succ }}$. Consequently both equations have to be analyzed to establish the conditions under which $P_{P, \text { succ }} \geq P_{D \text {,succ }}$. The following theorem establishes the condition under which there exists an efficient range of values for $p$ of the form $\left[0, p_{\max }\right]$, for some $0 \leq p_{\max }<1$, such that $P_{P, \text { succ }} \geq P_{D, \text { succ }}$.

Theorem 2. Provided that $\sum_{\forall u \in V}\left(\left|R_{u \rightarrow v}\right|-\left(q-\left|C_{u \rightarrow v}\right|\right)\left|S_{v}\right|\right) \geq 0$ is satisfied, there exist an efficient range of values for $p$ of the form $\left[0, p_{\max }\right]$, for some $0 \leq p_{\max }<1$.

Proof. The first derivative $\frac{d P_{P, s u c c}}{d p}$ is given by the following equation.

$$
\frac{d P_{P, s u c c}}{d p}=\frac{1}{N} \sum_{\forall u \in V} \frac{\left|R_{u \rightarrow v}\right|-\left(q-\left|C_{u \rightarrow v}\right|\right)\left|S_{v}\right|-\left|R_{u \rightarrow v}\right|\left(\left|S_{v}\right|+1\right) p}{q^{2}}(1-p)^{\left|S_{v}\right|-1} .
$$

It is obvious that for small values of $p$ (close to 0 but positive) $\lim _{p \rightarrow 0} \frac{d P_{P, s u c c}}{d p}=\frac{1}{N} \sum_{\forall u \in V} \frac{\left|R_{u \rightarrow v}\right|-\left(q-\left|C_{u \rightarrow v}\right|\right)\left|S_{v}\right|}{q^{2}}$. Consequently, if $\sum_{\forall u \in V}\left(\left|R_{u \rightarrow v}\right|-\left(q-\left|C_{u \rightarrow v}\right|\right)\left|S_{v}\right|\right) \geq 0$ there exists a range of values for $p$ such that $\frac{d P_{P, s u c c}}{d p} \geq 0$ and since $P_{P, \text { succ }}=P_{D, s u c c}$, for $p=0$, it is concluded that $P_{P, s u c c} \geq P_{D, \text { succ }}$; since for some $p \geq 0, P_{P, \text { succ }}$ is a continuous function of $p$ (see Equation (6)), and since $P_{P, s u c c}=0$, for $p=1$, there exists a value for $p$, denoted by $p_{\max }$, such that $P_{P, \text { succ }}=P_{D, \text { succ }}$ and $0 \leq p_{\max }<1$. Consequently, if the particular condition is satisfied then there exists an efficient range of values of the form $\left[0, p_{\max }\right]$.

The following theorem is based on Theorem 2 for "large networks" ( $k>1$, see [7]).

Theorem 3. For $k>1$, there always exists an efficient range of values for $p$ of the form $\left[0, p_{\max }\right]$, for some $0 \leq p_{\max }<$ 1, for which $P_{P, \text { succ }} \geq P_{D, \text { succ. }}$.

The proof of Theorem 3 can be found in Appendix 2.

Theorem 2 and Theorem 3 establish the conditions for the existence of an efficient range of values for the access probability $p$ of the form $\left[0, p_{\max }\right]$, for some $0 \leq p_{\max }<1$. This does not necessarily imply that there are no other efficient values of $p$ outside $\left[0, p_{\max }\right]$. Since $P_{P, s u c c}$ is a polynomial of degree $D+1$ (see Equation $(6)$ ), $\frac{d P_{P, s u c c}}{d p}$ is a polynomial of degree $D$ (see Equation (7)) and may have up to $D$ real roots. That is, $P_{P, \text { succ }}$ may assume up to $D$ extreme values. These extreme values correspond to a sequence of local maximum values immediately followed by a local minimum value and so on. Consequently, $P_{P, \text { succ }}$ may occasionally become greater or smaller than $P_{D, s u c c}$ as $p$ increases. Given that Equation (6) and Equation (7) are difficult or impossible to be analyzed for $D>1$ and $D>2$, respectively, any other value of $p \notin\left[0, p_{\max }\right]$ cannot be determined. Furthermore, the maximum value of $P_{P, \text { succ }}$ cannot be determined because the value of $p$ that achieves it cannot be determined as well. In addition, the range [0, $\left.p_{\text {max }}\right]$ cannot be determined analytically.

In order to avoid the aforementioned problems - (a) existence of a value of $p \notin\left[0, p_{\max }\right]$ for which $P_{P, \text { succ }} \geq P_{D, \text { succ }}$; (b) maximum value of $P_{P, s u c c}$; (c) analytical expression for $p_{\max }$ - an approximate analysis is considered and presented in the following section. This analysis establishes the appropriate conditions for the existence of an efficient range of values, based on a topology density metric. The maximum value for the system throughput is possible to be calculated, since the corresponding value of $p\left(\tilde{p}_{0}\right)$ is analytically determined. The boundaries for $\tilde{p}_{0}$ are also determined analytically as a function of the introduced topology density metric.

\section{Approximate Analysis}

The problems mentioned in the previous section arise from the polynomial nature of Equation (6) that is difficult or impossible to be analyzed for $D>2$. The approximate analysis presented in this section is based on a polynomial that is more tractable than that in Equation (6). Let the system throughput $P_{P, s u c c}$, be approximated by $\tilde{P}_{P, s u c c}$ :

$$
\tilde{P}_{P, s u c c}=\frac{1}{N} \sum_{\forall u \in V} \frac{q-\left|C_{u \rightarrow v}\right|+p\left|R_{u \rightarrow v}\right|}{q^{2}}(1-p)^{\overline{|S|}}
$$


where $\overline{|S|}=\frac{1}{N} \sum_{\forall u \in V}\left|S_{u}\right|$, denotes the average number of neighbor nodes. Let $\overline{|S|} / D$ be referred to as the topology density. For a given pair of $N$ and $D$, numerous topologies exist that can be categorized according to the average number of neighbor nodes. In the sequel, the conditions under which $\tilde{P}_{P, s u c c} \geq P_{D, s u c c}$, are established and the value for $p$ (denoted by $\tilde{p}_{0}$ ) that maximizes $\tilde{P}_{P, s u c c}$ is determined as well.

Let $\phi_{u \rightarrow v}=\frac{\sum_{\chi \in S_{v} \cup\{v\}-\{u\}}\left|\Omega_{\chi} \cap \Omega_{u}\right|}{\left|S_{v}\right|+1}$ denote the average number of overlapping slots of node $u$ with each node $\chi \in$ $\left(S_{v} \cup\{v\}-\{u\}\right)$. As it can be seen from Appendix 3, the following inequality holds.

$$
\left|R_{u \rightarrow v}\right| \geq q^{2}-\left(\left|S_{v}\right|+1\right)\left(q-\phi_{u \rightarrow v}\right) .
$$

Let $\bar{\phi}=\frac{1}{N} \sum_{\forall u \in V} \phi_{u \rightarrow v}$.

Theorem 4. Provided that $\sum_{\forall u \in V}\left(\left|R_{u \rightarrow v}\right|-\left(q-\left|C_{u \rightarrow v}\right|\right) \mid \overline{S \mid}\right) \geq 0$ is satisfied, there exists a range of efficient values of $p$ of the form $\left[0, \tilde{p}_{\text {max }}\right]$, for some $0 \leq \tilde{p}_{\text {max }}<1$. $\tilde{P}_{P, \text { succ }}$ assumes a maximum for $p=\frac{\sum_{\forall u \in V}\left(\left|R_{u \rightarrow v}\right|-\left(q-\left|C_{u \rightarrow v}\right|\right) \mid \overline{S \mid}\right)}{\sum_{\forall u \in V}\left(\left|R_{u \rightarrow v}\right|(\overline{S \mid}+1)\right)}$ $\left(\equiv \tilde{p}_{0}\right)$.

The proof of Theorem 4 can be found in Appendix 4.

Theorem 4 not only establishes the conditions for the existence of an efficient range of values for $p$, but also determines the value of $p\left(\tilde{p}_{0}\right)$ that maximizes $\tilde{P}_{P, s u c c}$. This is rather useful, but in the general case, knowledge of $C_{u \rightarrow v}$ and $R_{u \rightarrow v}$ for all possible transmissions in the network, is not available. Theorem 5 establishes a condition equivalent to that of Theorem 4 , based on the average number of overlapping slots $\bar{\phi}$ and the topology density $\overline{|S|}$. In addition, Theorem 6 determines the lower and upper bounds of $\tilde{p}_{0}$ as a function of $\overline{|S|}$ only.

Theorem 5. There exists an efficient range of values for $p$, provided that $\bar{\phi} \geq \frac{2 \overline{S \mid}+1}{4}$.

Proof. According to Theorem 4, there exists an efficient range of values of $p$ if $\sum_{\forall u \in V}\left(\left|R_{u \rightarrow v}\right|-\left(q-\left|C_{u \rightarrow v}\right|\right) \overline{|S|}\right) \geq 0$ holds. From Equation (9), $\left|R_{u \rightarrow v}\right| \geq q^{2}-(\overline{|S|}+1)\left(q-\phi_{u \rightarrow v}\right)$. Therefore, $\sum_{\forall u \in V}\left(\left|R_{u \rightarrow v}\right|-\left(q-\left|C_{u \rightarrow v}\right|\right) \mid \overline{S \mid}\right) \geq 0$ always holds if $\sum_{\forall u \in V}\left(q^{2}-(\overline{|S|}+1)\left(q-\phi_{u \rightarrow v}\right)-\left(q-\left|C_{u \rightarrow v}\right|\right) \overline{S \mid}\right) \geq 0$. Given that $\left|C_{u \rightarrow v}\right| \geq \phi_{u \rightarrow v}$ (see Appendix 5), it is enough to show that $\sum_{\forall u \in V}\left(q^{2}-(\overline{|S|}+1)\left(q-\phi_{u \rightarrow v}\right)-\left(q-\phi_{u \rightarrow v}\right) \overline{|S|}\right) \geq 0$ or $\sum_{\forall u \in V}\left(q^{2}-(2 \mid \overline{S \mid}+1)\left(q-\phi_{u \rightarrow v}\right)\right) \geq 0$ or $\left.\sum_{\forall u \in V}\left(q^{2}-(2 \overline{|S|}+1) q+(2 \overline{|S|}+1) \phi_{u \rightarrow v}\right)\right) \geq 0$ or $N q^{2}-N(2 \overline{|S|}+1) q+(2 \overline{|S|}+1) \sum_{\forall u \in V} \phi_{u \rightarrow v} \geq 0$ or $q^{2}-(2 \overline{|S|}+$ 1) $q+(2 \overline{|S|}+1) \bar{\phi} \geq 0$.

Let $\Delta$ be equal to $(2 \overline{|S|}+1)^{2}-4(2 \overline{S \mid}+1) \bar{\phi}=(2 \overline{|S|}+1)(2 \overline{|S|}+1-4 \bar{\phi})$. For $\Delta \leq 0, q^{2}-(2 \mid \overline{S \mid}+1) q+(2 \overline{|S|}+1) \bar{\phi} \geq 0$. Since $2 \overline{|S|}+1>0$, in order for $\Delta \leq 0,2 \overline{|S|}+1-4 \bar{\phi} \leq 0$ should hold, or $\bar{\phi} \geq \frac{2 \mid \overline{S \mid}+1}{4}$.

The condition of Theorem 5 (or Theorem 4) is sufficient but not necessary in order for $\tilde{P}_{P, \text { succ }} \geq P_{D, s u c c}$. Notice also that these theorems do not provide for a way to derive $\tilde{p}_{\text {max }}$. In addition, $\tilde{p}_{0}$ depends on parameters that are difficult to know for the entire network. In the sequel, Theorem 6 not only provides for a range of efficient values for $p$ but also determines simple bounds $\left(\tilde{p}_{0_{\max }}, \tilde{p}_{0_{\min }}: \tilde{p}_{0_{\max }} \leq \tilde{p}_{0} \leq \tilde{p}_{0_{\min }}\right)$ on the values of $\tilde{p}_{0}$ (that maximizes $\tilde{P}_{P, \text { succ }}$ ) as a function of the simple topology density $\overline{|S|}$.

Theorem 6. $\tilde{p}_{0_{\text {max }}}=\frac{1}{\mid \overline{S \mid}+1}$, and $\tilde{p}_{0_{\min }}=\frac{q^{2}-(2 \overline{|S|}+1)\left(q-\frac{2 \overline{\mid S}+1}{4}\right)}{\left(q^{2}-(\overline{|S|}+1)\left(q-\frac{2 \bar{S}+1}{4}\right)\right)(\overline{|S|}+1)}$, provided that there exists an efficient range of values for $p$.

Proof. According to Theorem 4, there exists an efficient range of values of $p$, if $\sum_{\forall u \in V}\left(\left|R_{u \rightarrow v}\right|-\left(q-\left|C_{u \rightarrow v}\right|\right) \mid \overline{S \mid}\right) \geq 0$ holds. Then, $\tilde{p}_{0}=\frac{\sum_{\forall u \in V}\left(\left|R_{u \rightarrow v}\right|-\left(q-\left|C_{u \rightarrow v}\right|\right) \mid \overline{S \mid}\right)}{\sum_{\forall u \in V}\left(\left|R_{u \rightarrow v}\right|(\overline{S \mid}+1)\right)}=\frac{1}{(\overline{|S|}+1)}\left(1-\frac{\sum_{\forall u \in V}\left(q-\left|C_{u \rightarrow v}\right|\right) \mid \overline{S \mid}}{\sum_{\forall u \in V}\left|R_{u \rightarrow v}\right|}\right) \leq \frac{1}{|S|+1}$. Therefore, $\tilde{p}_{0_{\max }}=\frac{1}{\mid \overline{S \mid}+1}$. From Equation (9), $\left|R_{u \rightarrow v}\right| \geq q^{2}-(\overline{|S|}+1)\left(q-\phi_{u \rightarrow v}\right)$ and given that $\tilde{p}_{0}=\frac{1}{(\overline{|S|}+1)}\left(1-\frac{\sum_{\forall u \in V}\left(q-\left|C_{u \rightarrow v}\right|\right) \overline{S \mid}}{\sum_{\forall u \in V}\left|R_{u \rightarrow v}\right|}\right)$, it is concluded that $\tilde{p}_{0} \geq \frac{1}{(\overline{|S|}+1)}\left(1-\frac{\sum_{\forall u \in V}\left(q-\left|C_{u \rightarrow v}\right|\right) \overline{S S \mid}}{\sum_{\forall u \in V}\left(q^{2}-(\overline{|S|}+1)\left(q-\phi_{u \rightarrow v}\right)\right)}\right)=\frac{\sum_{\forall u \in V}\left(q^{2}-(\overline{|S|}+1)\left(q-\phi_{u \rightarrow v}\right)-\left(q-\left|C_{u \rightarrow v}\right|\right) \overline{S \mid}\right)}{\sum_{\forall u \in V}\left(q^{2}-(\overline{|S|}+1)\left(q-\phi_{u \rightarrow v}\right)\right)(\overline{|S|}+1)}$. Since 
$\left|C_{u \rightarrow v}\right| \geq \phi_{u \rightarrow v}\left(\right.$ Appendix 5), $\frac{\left.\sum_{\forall u \in V}\left(q^{2}-(\overline{|S|}+1)\left(q-\phi_{u \rightarrow v}\right)-\left(q-\mid C_{u \rightarrow v}\right)\right) \mid \overline{S \mid}\right)}{\sum_{\forall u \in V}\left(q^{2}-(\overline{S \mid}+1)\left(q-\phi_{u \rightarrow v}\right)\right)(\overline{|S|}+1)} \geq \frac{\sum_{\forall u \in V}\left(q^{2}-(\overline{|S|}+1)\left(q-\phi_{u \rightarrow v}\right)-\left(q-\phi_{u \rightarrow v}\right) \overline{|S|}\right)}{\sum_{\forall u \in V}\left(q^{2}-(\overline{S S}+1)\left(q-\phi_{u \rightarrow v}\right)\right)(\overline{|S|}+1)}$ and consequently, $\tilde{p}_{0} \geq \frac{\sum_{\forall u \in V}\left(q^{2}-(\overline{|S|}+1)\left(q-\phi_{u \rightarrow v}\right)-\left(q-\phi_{u \rightarrow v}\right) \overline{S \mid}\right)}{\sum_{\forall u \in V}\left(q^{2}-(\overline{|S|}+1)\left(q-\phi_{u \rightarrow v}\right)\right)(\overline{|S|}+1)}=\frac{\sum_{\forall u \in V}\left(q^{2}-(2 \overline{S \mid}+1)\left(q-\phi_{u \rightarrow v}\right)\right)}{\sum_{\forall u \in V}\left(q^{2}-(\overline{|S|}+1)\left(q-\phi_{u \rightarrow v}\right)\right)(\overline{|S|}+1)}$. Finally, $\tilde{p}_{0} \geq$ $\frac{q^{2}-(2 \mid \overline{S \mid}+1)\left(q-\frac{\sum_{\forall u \in V} \phi_{u \rightarrow v}}{N}\right)}{\left(q^{2}-(\overline{|S|}+1)\left(q-\frac{\left.\sum_{\forall u \in V}^{\phi_{u \rightarrow v}}\right)}{N}\right)(\overline{|S|}+1)\right.}=\frac{q^{2}-(2 \mid \overline{S \mid}+1)(q-\bar{\phi})}{\left(q^{2}-(\overline{|S|}+1)(q-\bar{\phi})\right)(\overline{|S|}+1)}=\tilde{p}_{m i n}^{\prime}(\bar{\phi})$. From Appendix 6 it is concluded that $\frac{d \tilde{p}_{m i n}^{\prime}(\bar{\phi})}{d \bar{\phi}}>$ 0 , is always positive and therefore, $\tilde{p}_{\min }^{\prime}(\bar{\phi})$ increases as $\bar{\phi}$ increases. Consequently, the minimum value for $\tilde{p}_{0}, \tilde{p}_{0_{\min }}$, corresponds to the minimum value of $\bar{\phi}$ for which there exists an efficient range of values for $p$. This value according to Theorem 5 , corresponds to $\bar{\phi}=\frac{2 \overline{|S|}+1}{4}$ and as a result, $\tilde{p}_{0_{\text {min }}}=\frac{q^{2}-(2 \mid \overline{S \mid}+1)\left(q-\frac{2 \overline{|S|}+1}{4}\right)}{\left(q^{2}-(\overline{|S|}+1)\left(q-\frac{2 \overline{|S|}+1}{4}\right)\right)(\overline{|S|}+1)}$.

Both Theorem 5 and 6 are important for the realization of a system that efficiently implements the Probabilistic Policy. Given a polynomial assignment that satisfies Theorem 5 , for a value of $p$ between $\tilde{p}_{0_{\min }}$ and $\tilde{p}_{0_{\max }}$, the achievable system throughput is close to the maximum. For the determination of $\tilde{p}_{0_{\min }}$ and $\tilde{p}_{0_{\max }}$ it is enough to have knowledge of the topology density $\overline{|S|}$.

\section{On the Accuracy of the Approximation}

The analysis presented in Section 4 has established the conditions under which $\tilde{P}_{P, \text { succ }} \geq P_{D, \text { succ }}$, as well as the range of values of $p$ for which $\tilde{P}_{P, \text { succ }}$ is maximized. This section presents the cases for which (a) $\tilde{P}_{P, \text { succ }}$ is close to $P_{P, \text { succ }}$ in the sense that there exists a small number $\varepsilon_{1}$ such that $\left|P_{P, \text { succ }}-\tilde{P}_{P, \text { succ }}\right| \leq \varepsilon_{1}$ and (b) if the condition, for which $\tilde{P}_{P, \text { succ }} \geq$ $P_{D, \text { succ }}$ holds, is satisfied, then $P_{P, \text { succ }} \geq P_{D, \text { succ }}$ holds as well. In particular, according to Theorem 4 , there exists an efficient range of values such that $\tilde{P}_{P, s u c c} \geq P_{D, s u c c}$, if $\sum_{\forall u \in V}\left(\left|R_{u \rightarrow v}\right|-\left(q-\left|C_{u \rightarrow v}\right|\right) \mid \overline{S \mid}\right) \geq 0$ is satisfied and according to Theorem 2, there exists an efficient range of values such that $P_{P, \text { succ }} \geq P_{D, \text { succ }}$, if $\sum_{\forall u \in V}\left(\left|R_{u \rightarrow v}\right|-\left(q-\left|C_{u \rightarrow v}\right|\right)\left|S_{v}\right|\right) \geq 0$ is also satisfied. This section presents the case for which the aforementioned conditions are close in the sense that there exists a small number $\varepsilon_{2}$ such that $\left|\sum_{\forall u \in V}\left(\left|R_{u \rightarrow v}\right|-\left(q-\left|C_{u \rightarrow v}\right|\right) \mid \overline{S \mid}\right)-\sum_{\forall u \in V}\left(\left|R_{u \rightarrow v}\right|-\left(q-\left|C_{u \rightarrow v}\right|\right)\left|S_{v}\right|\right)\right| \leq \varepsilon_{2}$. It is also shown that as $\varepsilon_{2}$ increases linearly, $\varepsilon_{1}$ increases exponentially. For the case where $\varepsilon_{1}$ is not small, it is possible that $\varepsilon_{2}$ is small and $P_{P, \text { succ }} \geq P_{D, \text { succ }}$ holds if the condition corresponding to $\tilde{P}_{P, \text { succ }} \geq P_{D, \text { succ }}$ is satisfied. In general, $\varepsilon_{1}$ reflects the accuracy of $\tilde{P}_{P, \text { succ }}$ while $\varepsilon_{2}$ reflects the accuracy of the conditions that if satisfied, $\tilde{P}_{P, s u c c} \geq P_{D, \text { succ }}$ holds.

First, it is enough to determine the cases when $\varepsilon_{1}$ is close to zero. From Appendix 7 it is concluded that $\mid P_{P, \text { succ }}-$ $\tilde{P}_{P, \text { succ }} \mid \leq \frac{1}{N} \sum_{\forall u \in V}\left(\frac{q-\left|C_{u \rightarrow v}\right|+p\left|R_{u \rightarrow v}\right|}{q^{2}}(1-p)^{\overline{|S|}}\left|(1-p)^{\left|S_{v}\right|-\overline{|S|}}-1\right|\right) \leq \varepsilon_{1}$. Let $\operatorname{Var}\{|S|\}=\frac{1}{N D} \sum_{v \in V}|\overline{|S|}-| S_{v}||$ be defined as the topology density variation. It is evident that as $\operatorname{Var}\{|S|\}$ increases, $\varepsilon_{1}$ increases exponentially. Consequently, $\tilde{P}_{P, \text { succ }}$ is a good approximation of $P_{P, \text { succ }}$, for rather small values of $\operatorname{Var}\{|S|\}\left(\varepsilon_{1} \rightarrow 0\right)$. In particular for a network for which for all nodes $v \in V,\left|S_{v}\right|=D, P_{P, \text { succ }}=\tilde{P}_{P, \text { succ }}(\operatorname{Var}\{|S|\}=0)$. If $\left|S_{v}\right| \neq D$ but $\left|S_{v}\right|=\left|S_{\chi}\right|, \forall v, \chi \in V-\{u\}$ (only for node $u \in V,\left|S_{u}\right|=D$ ), then $P_{P, \text { succ }} \approx \tilde{P}_{P, \text { succ }}$, when $N$ is large; for $N \rightarrow \infty$, $\lim _{N \rightarrow \infty} \operatorname{Var}\{|S|\}=0$ and consequently, $P_{P, \text { succ }}=\tilde{P}_{P, \text { succ }}\left(\varepsilon_{1}=0\right)$.

Second, the absolute difference between the two conditions is calculated to be equal to $\left|\sum_{\forall u \in V}\left(q-\left|C_{u \rightarrow v}\right|\right)\left(\overline{|S|}-\left|S_{v}\right|\right)\right| \leq$ $\sum_{\forall u \in V}\left(q-\left|C_{u \rightarrow v}\right|\right)|\overline{|S|}-| S_{v}|| \leq \varepsilon_{2}$. Consequently, as $\operatorname{Var}\{|S|\}$ approaches zero, $\varepsilon_{2}$ approaches zero linearly and consequently, the condition under which $\tilde{P}_{P, \text { succ }} \geq P_{D \text {,succ }}$ holds, approaches linearly the condition under which $P_{P, \text { succ }} \geq P_{D, \text { succ }}$ holds. On the other hand, as $\operatorname{Var}\{|S|\}$ increases, $\varepsilon_{2}$ increases linearly but $\varepsilon_{1}$ increases exponentially.

Let $p_{0}$ denote that value for $p$ that maximizes (global maximum) $P_{P, \text { succ }}$. Obviously, $\left.\frac{d P_{P_{s} \text { succ }}}{d p}\right|_{p=p_{0}}=0$. Equation (7) is a polynomial of degree $D$ and it is difficult or impossible to be solved to obtain an analytical form for $p_{0}$. It is obvious that for $\operatorname{Var}\{|S|\}=0, \tilde{p}_{0} \equiv p_{0}$ and therefore, $p_{0} \in\left(\tilde{p}_{0, \text { min }}, \tilde{p}_{0, \text { max }}\right)$. In general $p_{0}$ may or may not belong in $\left(\tilde{p}_{0, \text { min }}, \tilde{p}_{0, \text { max }}\right)$ but any value $p \in\left(\tilde{p}_{0, \min }, \tilde{p}_{0, \max }\right)$ for which $\tilde{P}_{P, \text { succ }} \geq P_{D, \text { succ }}$ holds and $\tilde{P}_{P, \text { succ }}$ is close to its maximum value, possibly (depending on the value of $\varepsilon_{2}$ ) leads to $P_{P, \text { succ }} \geq P_{D, \text { succ }}$ and it is possible (depending on the value of $\left.\varepsilon_{1}\right)$ that $P_{P, \text { succ }}$ is close to its maximum value as well. 


\section{Simulation Results}

For the simulation purposes four different topology categories are considered. The number of nodes in each topology category is $N=100$, while $D$ is set to $5,10,15$ and 20. These four topology categories are denoted as D5N100, D10N100, D15N100 and D20N100 respectively. The algorithm presented in [7] that maximizes the minimum guaranteed throughput, is used to derive the sets of scheduling slots. Time slot sets are assigned randomly to each node, for each particular topology. The particular assignment is kept the same for each topology category throughout the simulations. Three different topologies that correspond to different topology density values $\overline{|S|} / D$ are considered for each topology category.
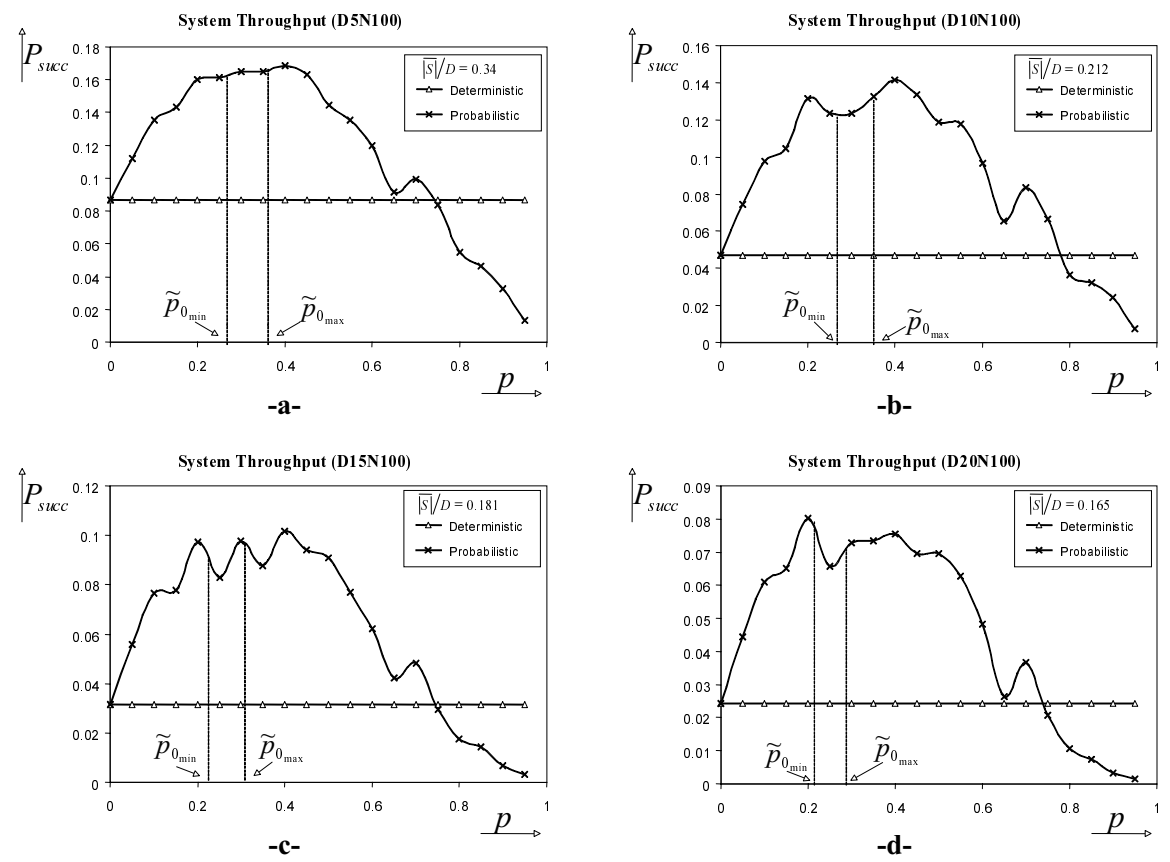

Fig. 1. System throughput simulation results for different values of $p$ (small topology density values $\overline{|S|} / D$ ), for both the Deterministic and the Probabilistic Policy.

According to Theorem 1, for $k>1$ the number of non-assigned eligible time slots is expected to be higher for a higher value of $k$ and there exists an efficient range of values for $p$, as it has been proved in Theorem 3. Consequently, it is expected that for $k>1$ all transmissions will achieve a higher system throughput under the Probabilistic Policy for any value of $p$ within the efficient range. When $k=1$, the Probabilistic Policy will outperform the Deterministic Policy if the condition of Theorem 5 is satisfied and $p$ belongs in the efficient range of values. If $p \in\left(\tilde{p}_{0, \min }, \tilde{p}_{0, \max }\right)$ then the achieved system throughput is possible to be close to the maximum, as it appears from Theorem 6 .

The simulation results presented demonstrate the performance for $k=1$ (the resulting value for $k$ is equal to 1 for the four topology categories, [7]), that is the case that the number of non-assigned eligible time slots is expected to be rather small and, thus, the effectiveness of the Probabilistic Policy rather low. The value of $\bar{\phi}$ calculated for each topology satisfies the condition of Theorem 5 for all cases.

Figures 1, 2 and 3 depict simulation results for the system throughput $\left(P_{\text {succ }}\right)$, under both the Deterministic and the Probabilistic Policies, as a function of the access probability $p$. In Figure $1, \overline{|S|} / D$ is small (around 0.2), in Figure 2, $\overline{|S|} / D$ is around 0.6, while in Figure 3, $\overline{|S|} / D$ is high (around 0.85). For all cases the value for the topology density variation $\operatorname{Var}\{|S|\}$ is the highest possible since the number of nodes, for which the number of neighbors is equal to $D$, is as high as possible and the rest of the nodes have the minimum number of neighbor nodes required (in order to allow connectivity).

In all three sets of simulations it can be observed, as expected, that the system throughput achieved under the Deterministic Policy is constant with respect to $p$. On the other hand, the system throughput under the Probabilistic Policy is equal to that under the Deterministic Policy for $p=0$ and equal to zero for $p=1$, as it may also be concluded from Equation (6). It can be observed that there exists an efficient range of values for the access probability $p$ for all cases. The range of values $\left(\tilde{p}_{0_{\text {min }}}, \tilde{p}_{0_{\text {max }}}\right)$, as it is determined by Theorem 6 , is shown as well. Obviously, $\left(\tilde{p}_{0_{\text {min }}}, \tilde{p}_{0_{\text {max }}}\right)$ determines a range of the values of $p$ for which $P_{P, s u c c}>P_{D, s u c c}$ and it appears that $P_{P, s u c c}$ is close to its maximum value.

For the comparison between the two schemes, it is set $p=\tilde{p}_{0_{\min }}$. From Figure 4 , it can be seen that the achieved system throughput under the Probabilistic Policy is higher than that under the Deterministic Policy. In particular, for small 

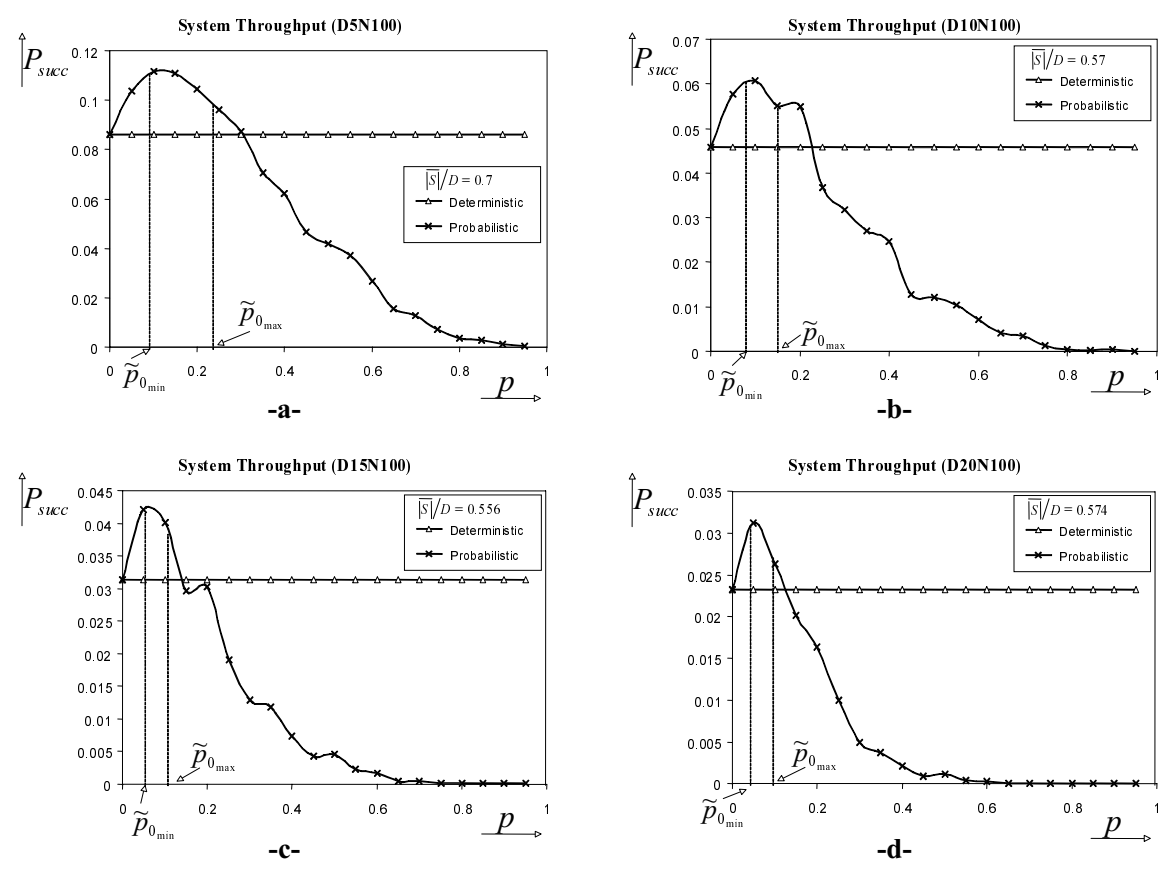

Fig. 2. System throughput simulation results for different values of $p$ (medium topology density values $\overline{|S|} / D$ ), for both the Deterministic and the Probabilistic Policy.

values of the topology density $\overline{|S|} / D$, the system throughput is almost double compared to that of the Deterministic Policy. As $\overline{|S|} / D$ increases the system throughput under the Probabilistic Policy converges to that under the Deterministic Policy. From Figure 4 it can also be observed that the achievable system throughput under the Probabilistic Policy decreases exponentially as the topology density $\overline{|S|} / D$ increases. This is also concluded from Equation (6).

\section{Summary and Conclusions}

The Probabilistic Policy was introduced in [8] and the throughput for a specific transmission was studied. In this work, this policy is considered and a system throughput analysis is presented in order to (a) identify the suitable range of values for the access probability $p$ for which the Probabilistic Policy outperforms the Deterministic Policy; (b) identify the maximum value for the system throughput and the corresponding value of the access probability; (c) determine simple bounds on the access probability that maximize the system throughput as a function of the topology density.

According to this analysis, even though the conditions for an efficient range of values of $p$ of the form $\left[0, p_{\max }\right]$ are established, it is shown that further analysis of the system throughput is difficult or impossible for certain cases. In particular, (a) $p_{\max }$ could not be determined analytically; (b) the maximum value of the system throughput could not be calculated as well as the corresponding value of the access probability $p$; (c) other existing values of $p$ such that $p \notin\left[0, p_{\max }\right]$, where the performance under the Probabilistic Policy is higher than that under the Deterministic Policy, could not be determined. The aforementioned problems motivated the continuation of the analysis based on certain approximations. An approximate analysis establishes the conditions under which the achieved system throughput is not only higher than that achieved under the Deterministic Policy, but it is also maximized. This analysis determines all values for $p$ such that the Probabilistic Policy outperforms the Deterministic Policy and the maximum value for the system throughput under the former policy can be analytically derived since the corresponding value of the access probability $p$ is analytically expressed. In addition, bounds on the latter value of $p$ are determined based on a the topology density metric that is possible to be known in the general case.

Simulation results have been derived for four network topology categories (for four pairs $(N, D)$ ) and for three values of the topology density $\left(\left|S_{v}\right| / D\right)$ for each topology category; $k=1$ and heavy traffic conditions have been assumed, both of which are expected to induce only a small advantage of the Probabilistic Policy over the Deterministic Policy, compared to the cases of networks with $k>1$ and non-heavy traffic conditions. The derived results have supported the claims and expectations regarding the comparative advantage of the Probabilistic Policy over the Deterministic Policy and that the approximate system analysis determines the range of values that, under certain conditions, maximize the system throughput under the Probabilistic Policy, or induce a system throughput close to the maximum. 

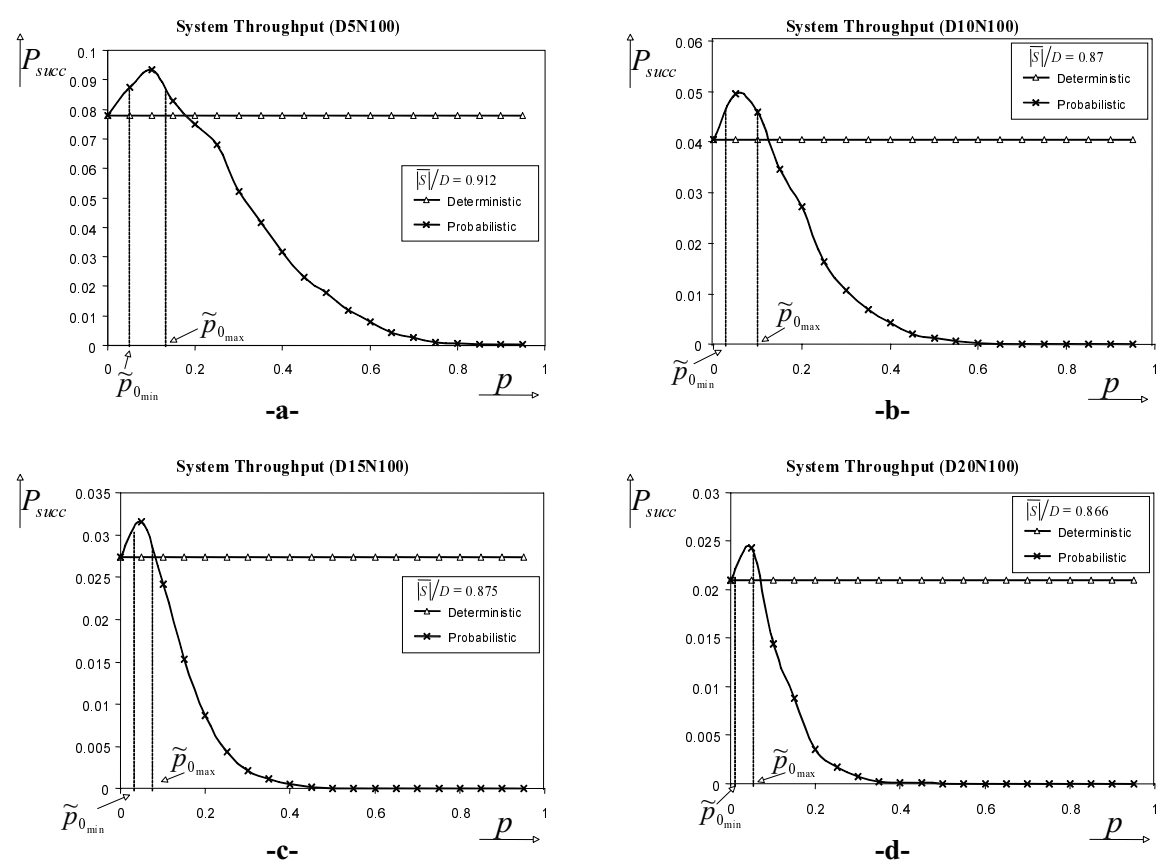

Fig. 3. System throughput simulation results for different values of $p$ (high topology density values $\overline{|S|} / D$ ), for both the Deterministic and the Probabilistic Policy.

\section{Acknowledgement}

All partners of the BROADWAY (IST-2001-32686) project are acknowledged. These are Motorola Labs, France, INTRACOM and University of Athens, Greece, TNO-FEL, Netherlands, IMST, IRK and Dresden University of Technology, Germany and FARRAN, Ireland.

The BROADWAY project is partly funded by the Commission of the European Union.

\section{References}

1. IEEE 802.11, "Wireless LAN Medium Access Control (MAC) and Physical Layer (PHY) specifications," Nov. 1997. Draft Supplement to Standard IEEE 802.11, IEEE, New York, January 1999.

2. V. Bharghavan, A. Demers, S. Shenker, and L. Zhang, "MACAW: A Media Access Protocol for Wireless LAN's," Proceedings of ACM SIGCOMM'94, pp. 212-225, 1994.

3. C.L. Fullmer, J.J. Garcia-Luna-Aceves, "Floor Acquisition Multiple Access (FAMA) for Packet-Radio Networks," Proceedings of ACM SIGCOMM'95, pp. 262-273, 1995.

4. P. Karn, "MACA- A new channel access method for packet radio," in ARRL/CRRL Amateur Radio 9th Computer Networking Conference, pp. 134-140, 1990.

5. J. Deng and Z. J. Haas, "Busy Tone Multiple Access (DBTMA): A New Medium Access Control for Packet Radio Networks," in IEEE ICUPC'98, Florence, Italy, October 5-9, 1998.

6. I. Chlamtac and A. Farago, "Making Transmission Schedules Immune to Topology Changes in Multi-Hop Packet Radio Networks," IEEE/ACM Trans. on Networking, 2:23-29, 1994.

7. J.-H. Ju and V. O. K. Li, "An Optimal Topology-Transparent Scheduling Method in Multihop Packet Radio Networks," IEEE/ACM Trans. on Networking, 6:298-306, 1998.

8. Konstantinos Oikonomou and Ioannis Stavrakakis, "A Probabilistic Topology Unaware TDMA Medium Access Control Policy for Ad-Hoc Environments," submitted to a conference.

\section{Appendices}

\section{Appendix 1 Proof of Theorem 1}

Notice that $\left|\bigcup_{\chi \in S_{v} \cup\{v\}} \Omega_{\chi}\right| \leq\left(\left|S_{v}\right|+1\right) q$, since $\left|\Omega_{\chi}\right|=q, \forall \chi \in V$. From Equation (2) it is concluded that $\left|R_{u \rightarrow v}\right| \geq$ $q^{2}-\left(\left|S_{v}\right|+1\right) q$, or $\left|R_{u \rightarrow v}\right| \geq q\left(q-\left|S_{v}\right|-1\right)$. Given that $D \geq\left|S_{v}\right|,\left|R_{u \rightarrow v}\right| \geq q(q-D-1)$. Since $q \geq k D+1$ (see [6], $[7]), q-D-1 \geq(k-1) D$. Consequently, $\left|R_{u \rightarrow v}\right| \geq q(k-1) D$.

(This proof is included to help the review process. It also appears in [8]). 

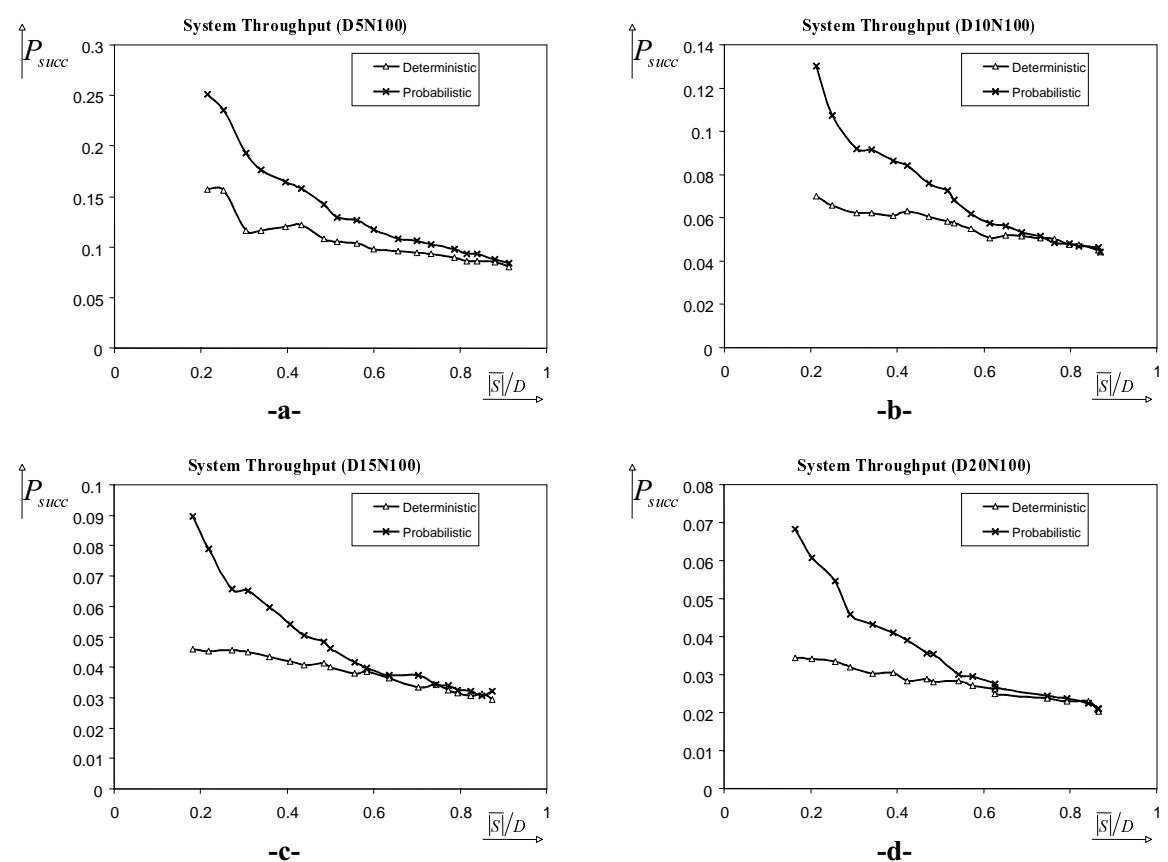

Fig. 4. System throughput simulation results $\left(P_{\text {succ }}\right)$, for both policies, for different values of the topology density $\overline{|S|} / D(p=$ $\left.\tilde{p}_{0_{\text {min }}}\right)$.

Appendix 2 Proof of Theorem 3

It is enough to show that $\sum_{\forall u \in V}\left(\left|R_{u \rightarrow v}\right|-\left(q-\left|C_{u \rightarrow v}\right|\right)\left|S_{v}\right|\right) \geq 0$ holds. From Theorem 1 and for any transmission $u \rightarrow v$, it is concluded that $\left|R_{u \rightarrow v}\right| \geq q D$, for $k>1$. Since $q \geq q-\left|C_{u \rightarrow v}\right|$ and $\left|S_{v}\right| \leq D$, it is concluded that $\left(q-\left|C_{u \rightarrow v}\right|\right)\left|S_{v}\right| \leq q D$, and, consequently, $\left|R_{u \rightarrow v}\right| \geq\left(q-\left|C_{u \rightarrow v}\right|\right)\left|S_{v}\right|$ holds for $k>1$, for every transmission $u \rightarrow v$. Finally, for $k>1, \sum_{\forall u \in V}\left(\left|R_{u \rightarrow v}\right|-\left(q-\left|C_{u \rightarrow v}\right|\right)\left|S_{v}\right|\right) \geq 0$.

(This proof is included to help the review process. It is partially based on a theorem included in [8]).

Appendix $3\left|R_{u \rightarrow v}\right| \geq q^{2}-\left(\left|S_{v}\right|+1\right)\left(q-\phi_{u \rightarrow v}\right)$

$\left|\bigcup_{\chi \in S_{v}+\{v\}} \Omega_{\chi}\right|$ can be written as $\left|\bigcup_{j=1}^{\left|S_{v}\right|+1} \Omega_{j}\right|$ by assigning numbers, $j=1, \ldots,\left|S_{v}\right|+1$, to each node $\chi \in S_{v} \cup\{v\}$.

Without loss of generality, it is assumed that node $u$ corresponds to number $\left|S_{v}\right|+1$ or $\Omega_{u} \equiv \Omega_{\left|S_{v}\right|+1}$.

$$
\begin{aligned}
& \left|\bigcup_{j=1}^{\left|S_{v}\right|+1} \Omega_{j}\right|=\left|\Omega_{1}\right|+\left|\bigcup_{j=2}^{\left|S_{v}\right|+1} \Omega_{j}\right|-\left|\Omega_{1} \cap\left(\bigcup_{j=2}^{\left|S_{v}\right|+1} \Omega_{j}\right)\right| \\
& \left|\bigcup_{j=2}^{\left|S_{v}\right|+1} \Omega_{j}\right|=\left|\Omega_{2}\right|+\left|\bigcup_{j=3}^{\left|S_{v}\right|+1} \Omega_{j}\right|-\left|\Omega_{2} \cap\left(\bigcup_{j=3}^{\left|S_{v}\right|+1} \Omega_{j}\right)\right| \\
& \left|\bigcup_{j=\left|S_{v}\right|-1}^{\left|S_{v}\right|+1} \Omega_{j}\right|=\left|\Omega_{\left|S_{v}\right|-1}\right|+\left|\bigcup_{j=\left|S_{v}\right|}^{\left|S_{v}\right|+1} \Omega_{j}\right|-\left|\Omega_{\left|S_{v}\right|-1} \cap\left(\bigcup_{j=\left|S_{v}\right|}^{\left|S_{v}\right|+1} \Omega_{j}\right)\right| \\
& \left|\bigcup_{j=\left|S_{v}\right|}^{\left|S_{v}\right|+1} \Omega_{j}\right|=\left|\Omega_{\left|S_{v}\right|}\right|+\left|\bigcup_{j=\left|S_{v}\right|+1}^{\left|S_{v}\right|+1} \Omega_{j}\right|-\left|\Omega_{\left|S_{v}\right|} \cap\left(\bigcup_{j=\left|S_{v}\right|+1}^{\left|S_{v}\right|+1} \Omega_{j}\right)\right| \\
& \left|\bigcup_{j=\left|S_{v}\right|+1}^{\left|S_{v}\right|+1} \Omega_{j}\right|=\left|\Omega_{\left|S_{v}\right|+1}\right| \text {. }
\end{aligned}
$$


Since $\left|\Omega_{j}\right|=q$, by adding all lines: $\left|\bigcup_{j=1}^{\left|S_{v}\right|+1} \Omega_{j}\right|=\left(\left|S_{v}\right|+1\right) q-\sum_{j=1}^{\left|S_{v}\right|}\left|\Omega_{j} \cap\left(\bigcup_{l=j+1}^{\left|S_{v}\right|+1} \Omega_{l}\right)\right|$. Let $\theta_{u \rightarrow v}=\frac{\sum_{j=1}^{\left|S_{v}\right|}\left|\Omega_{j} \cap\left(\bigcup_{l=j+1}^{\left|S_{v}\right|+1} \Omega_{l}\right)\right|}{\left|S_{v}\right|+1}$. The latter expression can be written as $\left|\bigcup_{j=1}^{\left|S_{v}\right|+1} \Omega_{j}\right|=\left(\left|S_{v}\right|+1\right) q-\left(\left|S_{v}\right|+1\right) \theta_{u \rightarrow v}=\left(\left|S_{v}\right|+1\right)\left(q-\theta_{u \rightarrow v}\right)$, and therefore, given Equation (2), $\left|R_{u \rightarrow v}\right|=q^{2}-\left(\left|S_{v}\right|+1\right)\left(q-\theta_{u \rightarrow v}\right)$.

$\phi_{u \rightarrow v}$ can be written as follows: $\phi_{u \rightarrow v}=\frac{\sum_{j=1}^{\left|S_{v}\right|}\left|\Omega_{j} \cap \Omega_{\left|S_{v}\right|+1}\right|}{\left|S_{v}\right|+1}$ or $\phi_{u \rightarrow v}=\frac{\sum_{j=1}^{\left|S_{v}\right|}\left|\Omega_{j} \cap \Omega_{u}\right|}{\left|S_{v}\right|+1}$. Given that $\Omega_{u} \equiv \Omega_{\left|S_{v}\right|+1}$, it is concluded that $\theta_{u \rightarrow v} \geq \phi_{u \rightarrow v}$ and consequently, Equation (9) is proved.

\section{Appendix 4 Proof of Theorem 4}

From Equation (8) it is concluded that $\tilde{P}_{P, \text { succ }}=0$ for $p=1$. Consequently, the range of values for which $P_{P, \text { succ }} \geq P_{D, \text { succ }}$ does not include 1 . On the other hand, for $p=0, \tilde{P}_{P, \text { succ }}=P_{D, \text { succ }}$ and therefore, 0 is included.

The first derivative of $\tilde{P}_{P, \text { succ }}$, with respect to $p$, is

$$
\frac{d \tilde{P}_{P, s u c c}}{d p}=\frac{1}{N} \sum_{\forall u \in V} \frac{\left|R_{u \rightarrow v}\right|-\left(q-\left|C_{u \rightarrow v}\right|\right)|\overline{S \mid}-| R_{u \rightarrow v} \mid(\overline{|S|}+1) p}{q^{2}}(1-p)^{\overline{S S \mid}-1} .
$$

For $0<p<1$ and $\sum_{\forall u \in V}\left(\left|R_{u \rightarrow v}\right|-\left(q-\left|C_{u \rightarrow v}\right|\right) \overline{S \mid}\right) \geq 0, \frac{d \tilde{P}_{P, s u c c}}{d p}=0$ when $p=\frac{\sum_{\forall u \in V}\left(\left|R_{u \rightarrow v}\right|-\left(q-\left|C_{u \rightarrow v}\right|\right) \mid \overline{S \mid}\right)}{\sum_{\forall u \in V}\left(\left|R_{u \rightarrow v}\right|(\overline{S S \mid}+1)\right)}$ $\left(\equiv \tilde{p}_{0}\right)$. From Equation (10) it is obvious that $\frac{d \tilde{P}_{P, s u c c}}{d p}>0$ for $p<\tilde{p}_{0}$ and $\frac{d \tilde{P}_{P, s u c c}}{d p}<0$ for $p>\tilde{p}_{0}$. Consequently, $P_{P, u \rightarrow v}$ assumes a maximum at $\tilde{p}_{0}$. Additionally, for every value $p, 0<p \leq \tilde{p}_{0}, \tilde{P}_{P, \text { succ }}>P_{D, \text { succ }}$.

For $p \rightarrow 1, \tilde{P}_{P, \text { succ }} \rightarrow 0$ and given that $\tilde{P}_{P, \text { succ }}$ is a continuous function of $p$, there exists a value $\tilde{p}_{\max }\left(\tilde{p}_{0}<\tilde{p}_{\max }<1\right)$ such that $\tilde{P}_{P, \text { succ }}=P_{D, \text { succ }}$. Finally, it is evident that for any value $p \in\left[0, \tilde{p}_{\text {max }}\right], \tilde{P}_{P, \text { succ }} \geq P_{D, \text { succ }}$, provided that $\sum_{\forall u \in V}\left(\left|R_{u \rightarrow v}\right|-\left(q-\left|C_{u \rightarrow v}\right|\right) \mid \overline{S \mid}\right) \geq 0$.

Appendix $5\left|C_{u \rightarrow v}\right| \geq \phi_{u \rightarrow v}$

From Equation (1), $\left|C_{u \rightarrow v}\right|=\left|\Omega_{u} \cap\left(\bigcup_{\chi \in S_{v} \cup\{v\}-\{u\}} \Omega_{\chi}\right)\right| \geq\left|\Omega_{u} \cap \Omega_{j}\right|$, for all nodes $\chi \in\left(S_{v} \cup\{v\}-\{u\}\right)$, denoted by numbers $j=1, \ldots,\left|S_{v}\right|$, while node $u$ is denoted by $\left|S_{v}\right|+1$. Consequently, $\left|S_{v}\right|\left|C_{u \rightarrow v}\right| \geq \sum_{j=1}^{\left|S_{v}\right|}\left|\Omega_{u} \cap \Omega_{j}\right|$, or $\left|C_{u \rightarrow v}\right| \geq \frac{\sum_{j=1}^{\left|S_{v}\right|}\left|\Omega_{u} \cap \Omega_{j}\right|}{\left|S_{v}\right|}>\frac{\sum_{j=1}^{\left|S_{v}\right|}\left|\Omega_{u} \cap \Omega_{j}\right|}{\left|S_{v}\right|+1}$, or $\left|C_{u \rightarrow v}\right|>\phi_{u \rightarrow v}$. Consequently, $\left|C_{u \rightarrow v}\right| \geq \phi_{u \rightarrow v}$ (the equality holds when $\Omega_{u} \cap\left(\bigcup_{\chi \in S_{v} \cup\{v\}-\{u\}} \Omega_{\chi}\right)=\emptyset$ in which case $\left.\left|C_{u \rightarrow v}\right|=\phi_{u \rightarrow v}=0\right)$.

Appendix $6 \frac{d \tilde{p}_{\min }^{\prime}(\bar{\phi})}{d \bar{\phi}}>0$.

$$
\begin{aligned}
\frac{d \tilde{p}_{\text {min }}^{\prime}(\bar{\phi})}{d \bar{\phi}} & =\frac{(2 \overline{|S|}+1)(\overline{|S|}+1)\left(q^{2}-(\overline{|S|}+1)(q-\bar{\phi})\right)-\left(q^{2}-(2 \overline{|S|}+1)(q-\bar{\phi})\right)(\overline{|S|}+1)^{2}}{(\overline{|S|}+1)^{2}\left(q^{2}-(\overline{|S|}+1)(q-\bar{\phi})\right)^{2}} \\
& =\frac{\overline{|S|} q^{2}}{(\overline{|S|}+1)\left(q^{2}-(\overline{|S|}+1)(q-\bar{\phi})\right)^{2}}>0 .
\end{aligned}
$$

Appendix $7\left|P_{P, s u c c}-\tilde{P}_{P, s u c c}\right|$

From Equation (6) and Equation (8), $\left|P_{P, \text { succ }}-\tilde{P}_{P, \text { succ }}\right|$ is calculated as follows.

$$
\begin{aligned}
\left|P_{P, \text { succ }}-\tilde{P}_{P, \text { succ }}\right| & =\left|\frac{1}{N} \sum_{\forall u \in V} \frac{q-\left|C_{u \rightarrow v}\right|+p\left|R_{u \rightarrow v}\right|}{q^{2}}(1-p)^{\left|S_{v}\right|}-\frac{1}{N} \sum_{\forall u \in V} \frac{q-\left|C_{u \rightarrow v}\right|+p\left|R_{u \rightarrow v}\right|}{q^{2}}(1-p)^{\overline{|S|}}\right| \\
& =\left|\frac{1}{N} \sum_{\forall u \in V} \frac{q-\left|C_{u \rightarrow v}\right|+p\left|R_{u \rightarrow v}\right|}{q^{2}}\left((1-p)^{\left|S_{v}\right|}-(1-p)^{\overline{|S|}}\right)\right|
\end{aligned}
$$




$$
\begin{aligned}
& \leq \frac{1}{N} \sum_{\forall u \in V}\left|\frac{q-\left|C_{u \rightarrow v}\right|+p\left|R_{u \rightarrow v}\right|}{q^{2}}\left((1-p)^{\left|S_{v}\right|}-(1-p)^{\overline{|S|}}\right)\right| \\
& =\frac{1}{N} \sum_{\forall u \in V} \frac{q-\left|C_{u \rightarrow v}\right|+p\left|R_{u \rightarrow v}\right|}{q^{2}}\left|(1-p)^{\left|S_{v}\right|}-(1-p)^{\overline{|S|}}\right| \\
& =\frac{1}{N} \sum_{\forall u \in V} \frac{q-\left|C_{u \rightarrow v}\right|+p\left|R_{u \rightarrow v}\right|}{q^{2}}(1-p)^{\overline{|S|}}\left|(1-p)^{\left|S_{v}\right|-\overline{|S|}}-1\right| .
\end{aligned}
$$

